Using Knowledge of Development to Promote Recovery of Function after Brain Damage

By: George F. Michel

This is the accepted version of the following article:

Michel, G. F. (2012). Using knowledge of development to promote recovery of function after brain damage. Developmental Psychobiology, 54(3) 350-356. doi: 10.1002/dev.20622,

which has been published in final form at http://dx.doi.org/10.1002/dev.20622

***@ $\subseteq$ Wiley. Reprinted with permission. No further reproduction is authorized without written permission from Wiley. This version of the document is not the version of record. Figures and/or pictures may be missing from this format of the document. $* * *$

\begin{abstract}
:
Knowledge of normal development of brain-behavior relations plays an important role in understanding how the plasticity of the nervous system can be used to promote recovery of function following brain damage. Aspects of the other articles in this issue are used in justification of the value of such developmental knowledge. Also, the development of amblyopia and its remediation in adulthood is discussed as a model for developing other techniques for ensuring recovery of function after stroke. Although the articles in this issue establish an excellent context for improving actual recovery of function (rather than compensation for deficits), much still needs to be discovered about how we can use developmental knowledge, along with knowledge of the plasticity of the nervous system, to improve remediation techniques.
\end{abstract}

Keywords: recovery of function | brain plasticity | ethology | critical period | amblyopia

Article:

\title{
INTRODUCTION
}

The Editor of this special issue set the tasks for the commentators to comment on how these articles investigating the processes involved in recovery of function after brain damage apply to amblyopia and how the research reported herein contributes toward "understanding developmental changes in plasticity more generally." Since I am not expert in the study of amblyopia and in order for me to undertake these tasks, I like to begin by first describing what I know about amblyopia and then placing that knowledge within the context of Tinbergen's (1963) proposal that explanations of the traits of animals (especially their behavior) reflect four mutually exclusive, but jointly complementary, modes of investigation (phylogenetic, adaptive, causal, and ontogenetic). Finally, I will address how the articles in this special issue address the relation between the plasticity processes in development and the plasticity processes involved in recovery of function after brain damage. 


\section{WHAT IS AMBLYOPIA?}

The normal development of binocular mechanisms in the visual cortex, thought to involve ocular dominance columns (ODC-alternating columns of cortical cells driven by input from the right and left eyes, respectively) requires precise matching of the images in the two eyes. Such interocular image matching depends on sets of connections (ODC) in the primary visual cortex (V1) of many primates that are present at birth (Horton \& Hocking 1996) and qualitatively adultlike soon after birth (Chino, Smith, Hatta, \& Cheng, 1997; Zhang et al.,2005). However, the continued functional development of these cortical binocular mechanisms critically depends on normal visual experience early in life (Mitchell \& Sengpiel, 2009). Any binocular imbalances (or interocular decorrelation of signals to the cortex) within a particular age range after birth (sensitive period) are known to cause binocular vision disorders or amblyopia.

Thus, amblyopia is traditionally considered to be a developmental disorder of spatial vision involving a difference in the clarity of input from the two eyes (because one has a cataract or is blurred by a refractive error) or an oculomotor control problem (that impairs concordance of the two retinal images). Such interocular disparity disrupts the normal maintenance of the ODC, with one eye (usually the more visually robust) usurping control over those columns previously driven by input from the "weaker" eye. More complex deficits in perceptual tasks that require spatial integration over a large area (such as contour integration) may also occur in amblyopia.

Experimental manipulations have demonstrated that amblyopia can be produced only if the differences between the eyes (e.g., monocular deprivation) are created at a particular point in the developmental process (the "critical" period). Similar perturbations later in development do not result in amblyopia. Moreover, brief periods of concordant binocular vision (as little as $30 \mathrm{~min}$ per day, Schwarzkopf et al., 2007) during the critical period can be sufficient to prevent the amblyopia created by the experimental interocular disparity (e.g., monocular deprivation).

In humans, amblyopia occurs in 2-4\% of the population and is almost always associated with an early history of abnormal visual experience between the two eyes such as binocular misregistration (strabismus); image degradation (high refractive error and astigmatism, and anisometropia); or form deprivation (congenital cataract and ptosis). Thus, the correlated visual signals from the two eyes to the visual brain depend on normal eye alignment, coordinated eye movements, and unrestricted vision in both eyes. These correlated signals are believed to be responsible for the maintenance of the cortical anatomical architecture relevant for binocular vision. The severity of amblyopia appears to be associated with the degree of imbalance between the two eyes and to the age at which the amblyogenic causes occurred. Different early visual experiences result in different functional losses in amblyopia (McKee, Levi, \& Movshon, 2003).

Maintenance of ODC by visual experience may depend on triggering of some biological events (such as gene expression) or simply allowing ODC to continue via some intrinsic fashion. In contrast, visual experience could sculpt development of the ODC according to the statistical 
character of the environment. If so, then amblyopia is a statistically unusual developmental event that depends on statistically "normal” or usual developmental processes. Evidence in support of a sculpting or instructive role of experience comes from studies that reared animals in environments with selective visual input (e.g., with only one eye open, Horton \& Hocking, 1997; or with each eye receiving a different set of visual cues for orientation, Hirsch \& Spinelli, 1970; Movshon \& Van Sluyters, 1981, or motion, Pasternak, Schumer, Gizzi, \& Movshon, 1985, or color, Sugita, 2004). Moreover, Dobkins, Bosworth, \& McCleery (2009) reported that the effects of visual experience may differ between the parvocellular (P) and the magnocellular (M) pathways. The M pathway may be more influenced by prenatal environmental developmental variables that are unrelated to visual experience whereas changes in the P pathway may depend on postnatal visual experience. Thus, amblyopia has stimulated much developmental research, that I will address later, but how do Tinbergen's other questions relate?

\section{PHYLOGENETIC HISTORY}

The phylogenetic history of binocular vision is important to determine because it greatly influences the interpretation of results from animal models. Phylogenetic history requires clear differentiation between homology (the identification of the relations among the animal model's neural architecture and functional organization that depend upon the relation of the model's evolutionary lineage to that of humans) and analogy (the identification of the relations among the model's neural architecture and functional organization that depend upon ecological constraints). Thus, the patterns of ocular dominance columns (ODC) in V1 cortex among different species can arise via common ancestry or via reactions to common ecological constraints. Homologous models can provide different information from analogous models about the trait's causation and development.

Examining the phylogenetic causes of any trait is intended to locate it in a genealogy of extinct and living organisms in order to determine ancestral features and patterns of relatedness among living species. This is represented in the typical comparative work of Ethology whereby attempts to identify homologies in the behavior of phylogenetically related species were used to infer the underlying ancestral behavioral traits that were believed to have been modified by evolutionary processes. Using ocular dominance columns (ODC) as the foundation for binocular vision, for example, would involve comparing their presence and function among current species of primates with that of humans in order to infer the ancestral patterns of hominids (indeed, all of the subgroups of the order—ape, old-world monkey, new world monkey, prosimian).

Such comparative investigation raises important issues for the selection of animal models. Using rats, cats, and ferrets as models presumes that the character of ODC is ancestral to mammals in general. However, there are too many exceptions in ODC patterns among species, even among primates (Adams \& Horton, 2009), and indeed among individual members of a species, for ODC to represent a mammalian ancestral pattern homologous with humans. If ODC is simply analogous between humans and their animal models, it increases the probability that the 
proximate causes and developmental processes may be fundamentally different, even if the adaptedness is similar. If ODC were not homologous among mammals, it would not be surprising then to discover that ODC do not require neural input from the eyes for ferrets but they do for macaques although macaques do not require visual experience whereas cats do (Adams \& Horton, 2009).

Although Tinbergen cautioned against mistaking phylogenetic homology as specifying the mechanisms and processes involved in the causation, development, and adaptive function of the trait, this caution applies more stringently to any analogous relationship. I will not have anything further to say about this mode of investigation for this commentary although issues of homology and analogy apply to any animal model used to identify the processes of recovery of function that will be relevant for humans. It is unfortunate that we cannot afford the comparative investigations needed to separate homologous and analogous relations among the animal models that we use for various human phenomena, including recovery of function from brain damage.

\section{ADAPTEDNESS}

The adaptedness of a trait is revealed by its effects on reproductive success. A trait may be important for the survival of the individual but it may only indirectly contribute to reproductive success. It is difficult to determine the adaptedness of binocular vision (its contribution to reproductive success) or even its function in humans. Certainly it contributes to depth perception and may promote important contour discriminations (valuable for humans when reading print) and distance perception (valuable for locomotion in trees). Binocular vision is more common in predators than in prey and that may be related to the need of prey to have a wider field of view to spot predators and the need of predators to more precisely "fix" the spatial location and distance of the prey. However, theories of vision and visual anatomy have not examined systematically the adaptedness of binocular vision. I will not have anything more to say about this mode of investigation in this commentary other than the following: Tinbergen warned against assuming that because a trait has a clear function that it automatically contributes directly to reproductive success, and hence was created by natural selection. Moreover, he also cautioned that the identification of the adaptedness of a trait did not specify either the mechanisms involved in its causation or the processes involved in its development.

\section{PROXIMATE CAUSATION}

Investigating the proximate causes of any species-typical trait involves examination of the biomechanical and physiological consequences of the individual's morphology and anatomy as these combine with the individual's social and physical context to affect the particular manifestation of the behavior at a particular time. Using binocular vision as the example again, this would involve identification of the neural mechanisms and biomechanical properties of the eyes governing visual functioning within a particular context that would include task demands (actions needed to successfully complete the task) and constraints (physical location of the task 
and the social milieu) that might affect binocular vision. I will say more about this mode of investigation later in my commentary.

\section{ONTOGENETIC HISTORY}

For Tinbergen, the ontogenetic history of a trait was represented by a series of transformations, during the lifespan of the organism, of the proximate mechanisms that create the developmental trajectory of the trait. In the case of binocular vision and its disturbance (amblyopia), this involves specification of the characteristics of the individual's neural (pattern of connections in the projections from the eye to cortex) and biomechanical (e.g., shape of the lens in the eyes) at one phase of development as these interact with the environmental contingencies (e.g., statistical patterns of photic information) to create emergent properties of neural organization at subsequent phases of development. From this perspective, it is possible to identify factors that perturb the trajectory as well as identify factors that potentially can remediate the trajectory. Thus, amblyopia is a developmental phenomenon that emerges from the effects of early visual experiences on the ODCs of the visual cortex that perturb the typical developmental trajectory. Knowledge of the factors governing the typical trajectory can be used to create remediation interventions that can help re-establish the more typical pattern. I will have more to say about this shortly.

As I have noted above, Tinbergen warned that the evidence generated by each of these modes of investigation should not substitute for one another. That is, phylogenetic or adaptation evidence is not a substitute for developmental or proximal evidence and developmental or adaptation evidence is not a substitute for proximate or phylogenetic evidence, etc. Rather, each of these modes of investigation deploys its own procedures and techniques and each provides a unique insight into the phenotypic character. Nevertheless, evidence from each of these modes of investigation can serve to prompt aspects of investigation in the other modes by making more salient potentially influential factors that otherwise might have been missed. Hence, these are four complementary modes of investigation. Nevertheless, I would argue that the developmental mode of investigation can provide a valuable perspective for the remediation of any trait when there has been a disruption of its normal proximate causal mechanism (e.g., recovery of function following brain damage).

Although the great evolutionary geneticist, Theodosius Dobzhansky, once opined that nothing in biology makes sense without the context of evolutionary theory, I would argue that developmental investigations make all aspects of biology more sensible. I agree with Dobzhansky that the manifestation of the vast variety of morphological and physiological characteristics of all living organisms becomes clearer when considered within their phylogenetic history (how modern organisms were related to one another via ancestry) and in relationship to the particular character of the natural selection forces operating within each species' ecological setting. I do not think that Dobzhansky meant that the investigation of phylogenetic history and adaptive significance of biological phenomena took precedence over 
proximal and developmental investigations. Rather, Dobzhansky might be interpreted as proposing that the phylogenetic and adaptive modes of investigation that were prompted by neoDarwinian evolutionary theory helped focus attention on proximate and developmental phenomena that otherwise might have been ignored or found to be confusing.

Likewise, I mean that recent work on developmental processes such as “epigenetics” (see WestEberhard, 2003; Michel, 2010) demonstrate how developmental investigations can reveal previously unnoticed issues in the proximate, adaptive, and phylogenetic investigation of phenotypic characters. That is, epigenetic mechanisms make transgenerational experiential and environmental influences possible without genetic modification. Moreover, they free theory from the notion that development is highly constrained by genetically determined programs.

In contrast to orthodox neo-Darwinian evolutionary theory, developmental investigation has revealed that the phenotypic characteristics manifest among members of a species are both constrained and created by complex patterns of environmental and genetic perturbations operating within the processes of development (c.f., Sansom \& Brandon, 2007). These developmental processes provide the phenotypic variety on which natural selection (along with other evolutionary processes such as drift, founder effects, etc.) operates to create the "new" morphological and physiological phenotypic characteristics. It is these developmentally created characteristics that mark the history of evolution and the adaptedness of the organism to its ecology. Consequently, modern biology has shifted focus to the investigation of how the organism's characteristics develop in an effort to identify the "rules” of developmental processes. That is an important shift for those interested in the ability of humans to recover functions (physiological and behavioral) lost to disease and accident because modern biology now seeks an understanding of the plasticity manifest in recovery of functions in the "plastic" processes that enable development.

The research reported in this special issue demonstrates the value that knowledge of the developmental processes contributes to the success of remediation procedures for recovery of psychological functions after damage to the nervous system. Development is an historical process in which previous events affect the manifestation of both current and subsequent events, and current events, in turn, become the previous events that affect subsequent events. Hence, there is a serial order to developmental phenomena that creates a trajectory of morphological and physiological phenotypes that can be individually specific while retaining species typicality (as noted in Kolb \& Teskey, this issue). Consequently, development should be defined by the illumination of the factors creating and governing the serial order of the trajectory and the processes that produce both change and stability of that order over time and across individuals (Michel \& Moore, 1995). Once developmental trajectories of particular phenotypes are specified, we can use these to discover how recovery might be accomplished.

Amblyopia provides both a good example of how disruptions in the trajectory of organismenvironment interaction can result in an anomalous phenotype and how knowledge of its 
development facilitates recovery. Although there is evidence of ODC in the visual cortex of primates before the typical pattern of neural activation from the two eyes occurs, particular perturbations in the young animal's exposure to light stimuli after birth, disrupts normal binocular vision and the maintenance of ODC (amblyopia). However, a critical or sensitive period for the occurrence of amblyopia should not be taken as evidence of a critical or sensitive period for recovery of function. The period is considered critical partly because it is impossible to create amblyopia by perturbations later in development and partly because it is more difficult to avoid or correct the amblyopia by manipulations later, than earlier, in development.

Although "age" appears to be the defining characteristic of the concept of critical period, central to its investigation is the recognition that there are specific events which must occur in a particular order for the typical development of subsequent characteristics to occur (Michel \& Tyler, 2005). Once the sequence of developmental events have occurred, it may be difficult to alter, compensate for, or reestablish the typical developmental trajectory and this leads to an atypical phenotype. Nevertheless, knowledge of the typical developmental trajectory of visual processing is essential for understanding what went wrong in the development of amblyopia and this knowledge may be used to identify clever ways of re-establishing a more typical trajectory and outcome later in the lifespan.

The work of Hensch (2005, this volume) and Castrén and colleagues (Maya-Vetencourt et al., 2008; Castrén \& Rantamäki, 2010) have demonstrated the importance of knowledge of developmental processes in promoting recovery of function in amblyopia. Hensch found that the critical period for amblyopia closes with the transition of cortical GABAnergic synapses from having excitatory properties to having inhibitory properties. Castrén and colleagues found that fluoxetine can reactivate developmental-like neuronal plasticity in the adult visual cortex (by increasing more generalized cortical excitation via prevention of the reuptake of serotonin), which, under appropriate environmental guidance, leads to the "rewiring" of a developmentally dysfunctional neural network and the loss of amblyopia. For those studies, amblyopia was created in rats by depriving one eye of visual input during the critical period. The recovery occurred in adults whose affected eye was kept open while the other eye was deprived of visual input during a chronic treatment of fluoxetine.

Castrén and Rantamäki (2010) propose that antidepressants like fluoxetine enhance serotonergic transmission, which promotes mechanisms that shift the intracortical inhibitory-excitatory balance (as described by Hensch), prompting plasticity in the adult visual cortex. The reduced GABAergic inhibition induced by chronic fluoxetine administration, and the increased expression of activity-induced increased BDNF (brain derived nerve growth factor) (an important contributor to the development of synaptic organization in the cortex), re-open the developmental pathways that regulate plasticity. Consequently, the neuronal circuitries that underlie the effects of monocular deprivation in the adult can be functionally modified, using knowledge about their development, and this leads to recovery from amblyopia, even in adulthood. The procedure of enforced use of the "weak" eye during the period of fluoxetine 
treatment is formally similar to that constraint-induced movement therapy proposed by Taub (this issue) for the recovery of motor functions after neural damage. Considering the importance of knowledge about the development of synaptic operations for the recovery from amblyopia, Taub's treatment might benefit from research that identifies the neurobiological processes that will prompt a reestablishment of the early developmental conditions of the synapses in those areas of the brain involved in the control of movements.

Similar to Taub's treatment, Levi and Li (2009) reported that severe amblyopia can be improved fivefold with 35,000 trials (about 50 hours) of perceptual learning and age does not seem to limit the efficacy of perceptual learning on improved visual functioning. Their procedure of perceptual learning provides intensive, active, supervised visual experience with feedback, and thus is quite different from, and consequently may be more effective than, reliance on everyday experiences (as occurs with simple occlusion treatment). During perceptual learning, observers are engaged in making fine judgments, with feedback, near the limit of their performance, using their amblyopic eyes (with their preferred eye occluded). Interestingly, there is transfer from the practiced tasks to performance on some other tasks such as letter acuity. Combining these results with those from the work of Hensch and Taub would indicate that some combination of the pharmacological manipulation of neurotransmitters and the imposition of perceptual learning techniques might be predicted to have the most benefit for recovery of function.

Kolb's and Teskey's (this issue) article reminds us of the principles of plasticity in the organization of the nervous system and emphasizes that there is much activity-generated and experience-dependent diversity in brain organization and function. They describe the complexity of the cascade of events that take place after cortical injury and they note the effects of age and sex on the processes of recovery. They report their research demonstrating the various ways by which recovery of function can be accomplished. Although the plastic changes in neuronal networks might be expected to be fairly widespread, they note that many experience-dependent changes are highly specific, both in location (both cortical region and cortical layer) and in the direction of the changes. Moreover, although the age-dependent nature of synaptic change is clearly important for understanding what treatments might be effective for pediatric versus adult neurological disorders, it is currently unknown what factors are responsible for this age dependency. Thus, they demonstrate the importance of obtaining knowledge of typical development for constructing appropriate remediation.

I would only add to their excellent account that perhaps, we should confine experiential influences to those requiring sensory activity (as proposed in Michel, 2010). Drugs, nutrition, and other environmental events need not operate by affecting sensory activity but nonetheless they can affect the development and recovery of function in the nervous system. Although experimental electromagnetic or electrical stimulation of the brain are not experiential events, they can be profoundly influential environmental manipulations of brain organization and functioning. Thus, Sharma and Cohen (this issue) demonstrate how transcranial magnetic stimulation (TMS) and transcranial direct current stimulation (tDCS) may be relevant to effective 
therapeutic treatment after stroke induced motor deficits. Also, Thompson, Mansouri, Koski, and Hess (this issue) use brain stimulation to offset the presumed imbalance of inhibitory and excitatory relations between the amblyopic and nonamblyopic eye's influence on V1 functional architecture to temporarily remediate amblyopia. Thus, these two articles demonstrate how important it is to consider and use nonexperiential, but environmental, events to promote recovery of function.

Since the concepts used to characterize neural development, neural plasticity, and recovery of function after neural damage share an affinity but nonetheless mark many different processes, we must be cognizant of these differences in order to avoid confusion and the inappropriate application of our knowledge. Neural development is marked by phenomena such as: multiple trajectories creating morphological and physiological variability both in the steps comprising the trajectory and in the endpoint of the trajectory; equifinality in which multiple trajectories converge on the same final endpoint; sequentially organized steps in which certain earlier phenotypes must be completed before subsequent phenotypes can occur (this sequential character is usually marked by critical periods in the developmental history); regression in which certain levels of organization are disrupted before developmental reorganization can occur.

As Corbetta (this issue) and Kolb and Teskey (this issue) note, neural plasticity in recovery of function is marked primarily by phenomena typically identified as learning and use-dependent organization (e.g., practice). Thus, recovery of function refers to the consequences of neural damage in which processes of learning and practice combine with developmental processes of multiple trajectories, regression, and the sequential dependency of transformations in phenotypic expression to constrain or promote the neural reorganization essential for either recovery of function or the establishment of compensatory functions.

Recovery of function typically refers to the changes in brain organization needed to compensate for a missing function and it involves both brain plasticity and developmental processes. Recovery can mean the re-establishment of lost abilities or skills (remediation) or the compensation of such loss by use of alternative strategies. The article by Small, Boccino, and Solodkin (this issue) remind us that, currently, too little rehabilitation with brain damaged patients focuses on remediation of deficits and instead focuses on ways to circumvent the deficits (compensation). Compensation is considered the most efficient way to achieve "recovery.” In compensatory recovery, different behaviors are used to meet environmental needs, and functional restoration is bypassed. In recovery through remediation, the lost behavior is actually restored, rather than circumvented. Because compensation is cheaper and quicker, it ends up the preferred approach for insurers, therapists, and often, patients. Nevertheless, Small et al. demonstrate how current knowledge of the mirror neuron system and its role in action understanding and imitation can serve as a means for promoting remediation in patients with stroke induced aphasia and upper limb deficits. 
Thus, the articles presented in this special issue create a need for the exploration of developmental processes that eventually can be applied to the recovery of function. There are similarities between the developing brain and the dynamic milieu of the adult injured brain. We should capitalize on these similarities when planning remediation procedures. Although the development of amblyopia can be a good model for some of this investigation, these articles demonstrate that knowledge about all developmental events have the potential for improving our treatment of patients suffering neural damage. We need to obtain information about which mechanisms for stability (e.g., hormonal condition, activity dependent neural circuits both intraand inter-hemispheric) make recovery from stroke more difficult in adults than in children. And we need to know how neural circuits for behaviors are established and maintained during development so that we can determine whether the circuits needed for recovery must be established and maintained in the same manner as they were during development. Fortunately, the articles presented in this issue point us in the right direction and move us further along the appropriate pathway. As Tinbergen might have noted, the information derived from developmental investigations is providing a context for examining the proximate causes operating after neural damage that can stimulate new therapies and better recovery.

\section{Acknowledgements}

While writing this article, George F. Michel's research was supported by NSF grant 0718045 .

\section{REFERENCES}

Adams, D. L., \& Horton, J. C. (2009). Ocular dominance columns: Enigmas and challenges. The Neuroscientist, 15(1), 62-77.

Castrén, E., \& Rantamäki, T. (2010). The role of BDNF and its receptors in depression and antidepressant drug action: Reactivation of developmental plasticity. Developmental Neurobiology, 70(5), 289-297.

Chino, Y. M., Smith, E. L. III, Hatta, S., \& Cheng, H. (1997). Postnatal development ofbinocular disparity sensitivity in neurons of the primate visual cortex. Neurosci 17, 296-307.

Corbetta, M. (this issue). Breakdown of functional connectivity and neurological recovery. Developmental Psychobiology.

Dobkins, K. R., Bosworth, R. G., \& McCleery, J. P. (2009). Effects of gestational length, gender, postnatal age, and birth order on visual contrast sensitivity in infants. Journal of Vision, 9(10), 19 (1-21).

Hensch, T. K. (2005). Critical period plasticity in local cortical circuits. Nature Reviews Neuroscience, 6, 877-888. 
Horton J. C., \& Hocking, D. R. (1996). An adult-like pattern of ocular dominancecolumns in striate cortex of new born monkeys prior to visual experience. Journal of

Neuroscience, 16, 1791-1807.

Horton, J. C., \& Hocking, D. R. (1997). Timing of the critical period for plasticity of ocular dominance columns in macaque striate cortex. Journal of Neuroscience, 1, 3684-3709.

Hirsch, H. V., \& Spinelli, D. N. (1970). Visual experience modifies distribution of horizontally and vertically oriented receptive fields in cats. Science, 168, 869-871.

Kolb, B., \& Teskey, G. C. (this issue). Age, experience, injury, and the changing brain. Developmental Psychobiology.

Levi, D. M., \& Li, R. W. (2009). Improving performance of the amblyopic visual system. Philosophical Transactions of the Royal Society B, 364, 399-407.

Maya-Vetencourt, J. F., Sale, A., Viegi, A., Baroncelli, L., De Pasquale, R., O'Leary, O. F. ... Maffei, L. (2008). The antidepressant fluoxetine restores plasticity in the adult visualcortex. Science, 320(5874), 385-388.

McKee, S. P., Levi, D. M., \& Movshon, J. A. (2003). The pattern of visual deficits in amblyopia. Journal of Vision, 3, 380-405.

Michel, G. F. (2010). The roles of environment, experience, and learning in behavioral development. In K. Hood, C. Halpern, G. Greenberg, \& R. Lerner (Eds.), Handbook of developmental science, behavior and genetics (pp 123-165). Malden, MA: Wiley.

Michel, G. F., \& Moore, C. L. (1995). Developmental psychobiology: An interedisciplinary science. Cambridge MA: The MIT Press.

Michel, G. F., \& Tyler, A. N. (2005). Critical period: A history of the transition from questions of when, to what, to how. Developmental Psychobiology, 46(3), 156-162.

Mitchell, D. E., \& Sengpiel, F. (2009). Neural mechanisms of recovery following early visual deprivation. Philosophical Transactions of the Royal Society B, 364, 383-398.

Movshon, J. A., \& Van Sluyters, R. C. (1981). Visual neural development. Annual Review of Psychology, 32, 477-522.

Pasternak, T., Schumer, R. A., Gizzi, M. S., \& Movshon, J. A. (1985). Abolition of visual cortical direction selectivity affects visual behavior in cats. Experimental Brain Research, 61, 214-217.

Sansom, R. \& Brandon, R. N. (Eds.), (2007). Integrating evolution and development: From theory to practice. Cambridge MA: The MIT Press. 
Schwarzkopf, D. S., Vorobyov, V., Mitchell, D. E., \& Sengpiel, F. (2007). Brief daily binocular vision prevents monocular deprivation effects in visual cortex. European Journal of Neuroscience, 25, 270-280.

Sharma, N., \& Cohen, L. G. (this issue). Recovery of motor function after stroke. Developmental Psychobiology.

Small, S.L., Boccino, G., \& Solodkin, A. (this issue). The mirror neuron system and treatment of stroke. Developmental Psychobiology.

Sugita, Y. (2004). Experience in early infancy is indispensable for color perception. Current Biology, 14, 1267-1271.

Taub, E. (this issue). Parallels between use of constraint-induced movement therapy to treat neurological motor disorders and amblyopia training. Developmental Psychobiology.

Thompson, B., Mansouri, B., Koski, L., \& Hess, R.F. (this issue). From motor cortex to visual cortex: The application of non-invasive brain stimulation to amblyopia. Developmental Psychobiology.

Tinbergen, N. (1963) On the aims and methods of Ethology. Zeitschrift fuer Tierpsychologie, 20, 410-433.

West-Eberhard, M. J. (2003). Developmental plasticity and evolution. Oxford: Oxford University Press.

Zhang, B., Zheng, J., Watanabe, I., Maruko, I., Bi, H., Smith, E. L. III, \& Chino, Y. (2005). Delayed maturation of receptive field center/surround mechanisms in V2. Proceedings of the National Academy of Sciences of the United States of America, 102,5862-5867. 